\title{
Query Optimization in a Blockchain-Based Land Registry Management System
}

\author{
Amrendra Singh Yadav*, Dharmender Singh Kushwaha \\ Computer Science and Engineering, Motilal Nehru National Institute of Technology Allahabad, Prayagraj 211004, India
}

Corresponding Author Email: amrendrayadav@mnnit.ac.in

https://doi.org/10.18280/isi.260102

Received: 14 November 2020

Accepted: 27 January 2021

\section{Keywords:}

peer-to-peer, blockchain, property transaction system, query optimization, consensus algorithm

\begin{abstract}
Digitization of land records is not sufficient for preventing fraud cases, time delay, and brokers' involvement. Distributed Ledger Technology (DLT) is used for making this digitized record more secure and process it in a decentralized way, and reduces paperwork in selling and buying of land. Blockchain technology has come to the fore in recent years and is the center of technical discussion, with intuitive applications driven by its network architecture. It has been firmly established as one of the most important emerging technologies. This article aims to implement a land registry mechanism using blockchain technology and optimize searching of land records in blockchain. Interplanetary File System (IPFS) provides an infrastructure that offers a precise portrayal of all the members' roles. The application interacts with the blockchain network, which is built using IPFS. This paper will help in providing a secure and decentralized system for the land registry process. The proposed consensus algorithm MRRCM achieves less time required to generated a block on an average by $3.06 \%$ round-robin and $96.48 \%$ PoW approach. The proposed modified hash table search approach requires less time to search the blockchain's land record block than the extensive liner searches and hash table search approach. A search for a land record in the blockchain reduces the search time on an average by $59.5 \%$ compared to the traditional extensive liner search approach and by $18.68 \%$ as compared with the hash table search approach.
\end{abstract}

\section{INTRODUCTION}

Expansion in technology has introduced incredible changes in commercial, industrial, academic, administration, etc. The involvement of technology is also required in the land registry sector in the real state industry. Whenever any land/ property is transferred from one entity to another entity, changing ownership is needed, called the land registration process. This process requires several steps to be performed between buyer and seller, such as stamp duties sale agreement, sale deed, and transfer deed. Stamp duty is a fee that has to be paid to the government to execute property transactions and related documents like conveyance deed, sale deed, and sale agreement [1]. The state-level authority governs the land registry process, and the sub-region specific authority to maintain and implement these policies in India. Every state has an independent system. It has no integration with other systems, which leads to inefficiency. Each authority establishes the rules and regulations that are necessary for the registration of the land registry. Registration charges and land rates may vary in different states. However, the land registration process is more or less the same across the country. With the advancement in information technology, some data relating to land register entries are available in digital form, others are being processed. Records such as land registry process, mutation, sale agreement, etc shell be digitized shortly. However, the problem of integrity and coordination remains. Numerous documents like Khasra, Khatauni, Shajra, Registration Document and Parwana could refer to the land record. Due to this improper definition, India has a massive amount of land dispute cases.
In India, the history of conflicts over property and the loss caused by these is evident. The latest study has revealed that investments worth about Rs 13.7 trillion (Rs 13.7 lakh crore) were embroiled in ongoing land conflicts across India, which, on average, impact about 10,600 people in every conflict. It is a concern for the country to enforce a property registry system to confront discrepancies arising with the present system.

In today's scenario, sellers and buyers meet at the registry office, share the physical documents in the registrar's presence, and complete the land registry registration process. Most of the transactions take place through the exchange and verification of physical copies. Many times various problems may occur during the land registry process, such as:

- The involvement of several intermediaries

-The increasing number of fraud cases

- Time Delays

-Human error and Intervention

-The problem of Forged Documents

-Loss of record

The Digital India National Land Records Modernization Programme (DNLRMP) was launched in 2008 [2]. It is a significant reform in this sector. The motive is to digitize all land records and fix inefficiencies through a survey of all lands. Digitization of property records, similar to the digitization of any other type of record, will improve transactions' efficiency by reducing the time taken to update or access records. However, it does not address trust deficiency arising out of complex processes and corrupt practices.

The conventional methods of storing land registries suffer from various severe issues due to that; the current system is not trusted in by the citizen. Many times citizens are unsure 
about the legality and authenticity of owned property and buying property, even if sellers have a legitimate deed of sale. Blockchain can fix many such problems [3]. Solutions based on DLT provide the immutable history of transaction records so that no one can ever doubt its authenticity. Land related data can be permanently connected to the authorized system so that nobody can ever manipulate or falsify their data. Any user can verify these records from web pages $24 * 7$.

The proposed work presents a framework of blockchain for the "Indian Land Registry System." Hence this work intends to create a solution that could bring all these land records under one system, and at the same time, maintain its integrity. This article provides a framework for decentralized, peer-to-peer, and safe infrastructure for handling land-related transactions. The records of properties are governed by decentralized organizations of the states/region with their websites. The application maintains and monitors these records by the interactions of the state/region peers. The ledger consists of the information on the property as well as the transaction history. We have implemented the blockchain-based system using the IPFS network. This aims at efficient functioning of the property ownership management organizations by storing, transferring, adding, and updating land information among different regions, maintaining consistency and security. The individuals or organizations can check the present status and the property ownership transfer history.

Blockchain technology provides a solution considering all the problems discussed with the traditional framework of property record maintenance. With its characteristics, a public user can also query the land registry management system to check out his/her ownership history without a need for an intermediary institution. An access control mechanism can also be enforced, not allowing unauthorized users to manipulate the information.

This article proposes a system that uses blockchain technology to store property transactions in an everlasting distributed ledger. Verifying the accuracy of the data is possible. The implementation of blockchain can also overcome human errors and delays. Hence, Distributed Ledger Technology (DLT) based blockchain solution is seeking the attention of researchers for the implementation of various financial and e-governance applications. In order to address these issues, we have proposed a blockchain based technology.

The rest of the article is organized as follows. Section 2 briefs the literature reviews related to the proposed work. Section 3 presents the proposed work and is divided into five subsections. The proposed consensus algorithm briefly explain in section 3.1. Section 3.2. presents the validation transaction module. The Add block to the blockchain method is explained in section 3.3. Section 3.4 proposes the view of the generation block module is valid or invalid. Sections 4 module is for the query ledger module explain three modules: extensive liner search, second hash table approach, and optimized hash table approach. Section 5 defines the attribute of a property transaction in block transfer ownership is transferred. Section 6 delivers comparative results and analyze them for the betterment of the proposed approach. Finally, section 7 concludes this article. The next section describes the literature reviews related to the proposed work, consensus mechanisms, and applications based on DLT.

\section{RELATED WORK}

Blockchain technology came into the picture when Nakamoto, in 2008, introduced bitcoin [4]. Nakamoto implemented it as the backbone of bitcoin in the cryptocurrency and served as the general ledger for all transactions in the network. By using a blockchain, bitcoin was the first digital currency to solve the double-spending problem. Blockchain, as the key technology behind bitcoin, is essentially a distributed database system. Zheng et al. [5] attempts to discuss blockchain architecture, consensus, and future trends. The blockchain works as an immutable ledger that permits transactions to occur in a decentralized manner. Blockchain-based applications are growing rapidly, covering numerous areas, including financial services, reputation systems, and the Internet of Things (IoT), and so on. However, blockchain technology has many challenges, such as scalability and security problems waiting to be overcome. Author a comprehensive overview of blockchain technology and compare some typical consensus algorithms such as Proof-of-Work (PoW), Delegated Proof of Stake (DPoS), Practical Byzantine Fault Tolerance (PBFT), and Proof-ofStake (PoS) used in blockchains. Furthermore, technical challenges and recent advances are briefly listed.

Some scholars implement a land register tool to create transparency in the land registry record [6]. The author provides secure time ordered, immutable process steps, configurable blockchain per missioning, integrity for files, and data store in a blockchain. Tschorsch and Scheuermann [7] state that the inspection process referred to as mining can be carried out through a work certificate, a batch test, or another mechanism. This facilitates the consensus under the node; otherwise, malicious entities could kidnap the consensus and destroy the system. Some scholars propose a ledger system tracking digital info that may enable people to collateralize property [8]. To increase the potential further, it is also partnered with a Visakhapatnam-based firm to handle land records.

Singh et al. [9] propose that public or private financial institutions worldwide reduce the time between purchase and payment, clearing, and settlement. Kumar et al. [10] propose validation a block and consensus mechanism, which generated a block randomly and added to the blockchain only if achieved $51 \%$ of nodes agree. Singh and Vardhan $[11,12]$ develop a Distributed Ledger Technology (DLT) based e-stamp procurement system. It is used for authentication for real estate documents such as stamp duty, property record, and ownership history. Mallick and Kushwaha [13] have propose an improved version of PUSH named Ex-PUSH protocol. The proposed publication-subscribe system can deliver the notification with content delivery capabilities to the subscriber. Singh and Vardhan [14] propose a decentralized and secure P2P cloud-based system to manage transaction capacity, increase network scalability, and optimize transaction and building time. Singh et al. have expanded the scope of electronic checks, including worldwide banking, and have also analyzed electronic checks' weaknesses, including doublespending and counterfeiting $[15,16]$. Singh and Vardhan [17] have proposed a framework decentralized architecture using blockchain to prevent fraudulent actions while allowing for a majority vote for each transaction and verification request. ChromaWay [18] has partnered with Andhra Pradesh's government to build a blockchain-powered system for land registration. The solution can eliminate fraud and errors and 
significantly reduce the administrative burden of land registration and title transfer. The solution provides value by improving processes associated with land registration and real estate transactions. Yadav et al. [19] propose a consensus mechanism for a land registry system to provide more secure and fast accessing of shared land record information and evaluate the performance of a blockchain-based implementation of land revenue, stamp duty, and recording automation system.

Nguyen and Kim [20] explain the PoW is one of the most popular consensus algorithms in the bitcoin cryptocurrency blockchain network. They accept the longest chain as the valid chain that depicts the system's historical transactions. Despite the proof-of-work success, it also has some limitations. One of the significant drawbacks of the proof-of-work consensus algorithm is its low throughput. Also, proof-of-work is susceptible to a $51 \%$ attack, which means if an attacker's hash computing power exceeds $51 \%$, they can control the network. Gupta et al. [21] employ block-transfer strategies to enhance transaction rates. Spectre uses a Directed Acyclic Graph (DAG) instead of the traditional "chain" structure, allowing more miners. The proof-of-work based consensus is vulnerable to attacks like DDOS attacks and stake grinding. It also creates the need for a new consensus protocol called PoS. Kiayias et al. [22] randomly select a subset of stakeholders as a contributor in an epoch. Proof-of-Stake consensus algorithm with security.

\section{PROPOSED WORK}

The traditional system of holding land registers suffers from severe problems in many countries across the globe. The majority of individuals do not believe in the existing conventional method. They are not entirely sure that they have all the land records, even though they have legal documents for the land record. People willing to buy land go into great pain to ensure that they are buying legitimately and are not cheated in the contract. By using blockchain, one can solve most of these problems. Therefore, this article's main goal is to establish a blockchain solution to address the traditional form of land registry documentation issues. The preservation of blockchain land registers offers us many significant advantages of an immutable record of all past transactions, thereby preserving validity. This is because once created, the data records stored in the blockchain cannot be modified. Thus, no one can fake the documents, and the records are also transparent so that anyone can check such records. To construct our blockchain using a distributed consensus mechanism, we use DLT. The blockchain system consists of peers linked by the peer network to the peer swarm. The blockchain can be reached by all the block in our blockchain network using a hash of blocks. A land registry system is linked to each state/region peer. There is a regional database for each land registry system. There is a list of land-IDs and their owners in the regional database. Thus, each peer must verify that the seller owns the land or not once a transaction occurs. Government officials would fully regulate the proposed land record management system. In the latest proposed blockchain system, all the existing government land records will then be stored. Using IPFS, we built the proposed blockchain framework. The government may also set up mining policy and consensus processes according to their needs.
This article proposes a scalable and novel property/land transaction framework to provide a fast, secure, transparent, and tamper-proof land registration system. The proposed framework is based on blockchain technology. Here we have used the InterPlanetary File System (IPFS) is a distributed and Peer-to-Peer (P2P) network, to implement our application shown in Figure 1.

Our proposed query search engine is built upon the collaboration of four main integrants, that are:

Lib P2P: It is a network framework that allows to writing the decentralized peer to peer applications.

IPFS storage: IPFS provides flexibility for the developers to develop their mining policies and consensus mechanisms for the blockchain application. IPFS platform has much functionality, such as content-addressable data sharing, P2P network, and decentralization.

Search Engine: Store and organize content during crawling and returns the result.

Summary File: It is a data management tool from where users perform query operations.

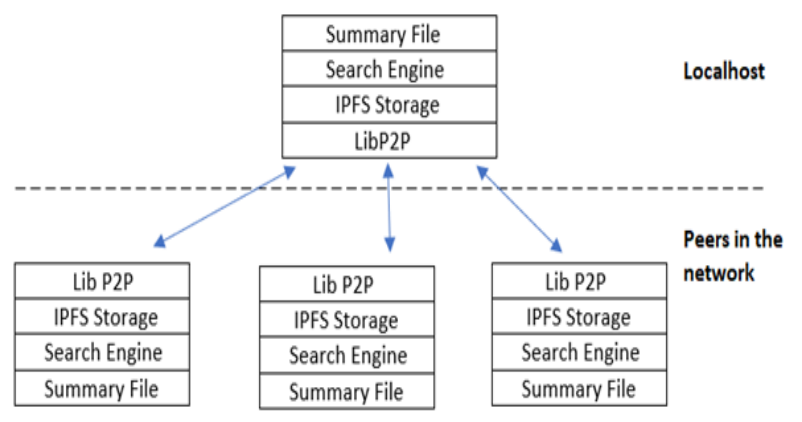

Figure 1. Peer-to-peer system architecture

The proposed architecture shall keep policies the same and only digitize and automate the processes. The complete transaction process is explained in the next subsection.

\subsection{Consensus mechanism}

A consensus mechanism is a process that is used between distributed systems to achieve an agreement on a single data value. Various researchers have proposed several consensus algorithms to achieve reliability in a network when there is a threat of unreliable nodes in the system, such as PoW, PoS, DPoS, etc. A consensus must be achieved before adding a new block into the blockchain. This article proposes a consensus mechanism named as Modified Round-robin Consensus Mechanism (MRRCM). The proposed method of consensus decreases the effort to add a new block, make it secure and synchronized. Synchronizing the general ledger of transactions over the network ensures that the ledger blocks are only updated when the corresponding participants approve the transactions, which is an essential activity. It can become part of the blockchain only for valid transactions. Validation is done only when a node in the network offers a guaranteed transaction ordering mechanism and validates the transaction block.

3.1.1 Proposed modified round-robin consensus mechanism (MRRCM)

This article proposes MRRCM that is an improved version of the round-robin consensus algorithm. The workflow of the proposed consensus mechanism is illustrated in Figure 2. The 
allot leader module runs in any one node of the blockchain network. This module is responsible for selecting the leader node. Leader nodes are allocated in a round-robin manner, and only the selected node is requested to add a new block. This means that first, the transaction values will be inputted by the user, then a request will be made to add a new block. Suppose there exist three nodes named A, B, and C in the network. The node $\mathrm{A}$ send two times and node $\mathrm{B}$ one time request at any given time, while node $\mathrm{C}$ did not send any request. Suppose node $\mathrm{A}$ is considered the first leader node, then it will add one new block. Now, node B will become the leader node and add a new block. Again node A is assigned as a leader node to add a second new block. But node $\mathrm{C}$ node is never considered as leader node because it did not send any request. Therefore, the MRRCM reduces the time during the addition of new blocks due to no involvement of node $\mathrm{C}$ during addition.

As shown in Figure 2, the transaction starts from the 'start' process node, and the transaction acts as input to 'make_block.' Now make block sends a request to 'allot leader_node' for allotment of the leader node, and leader is allocated according to the algorithm 1 . The leader node sends a validation proposal request to the 'validate' process to validate the proposal to add a new block. The validator node validates the transaction proposal request sent by the allocation leader. If the validator node receives a $51 \%$ majority of votes, then it responds to the leader node for the addition of a new block to the blockchain; Otherwise, the block is discarded.
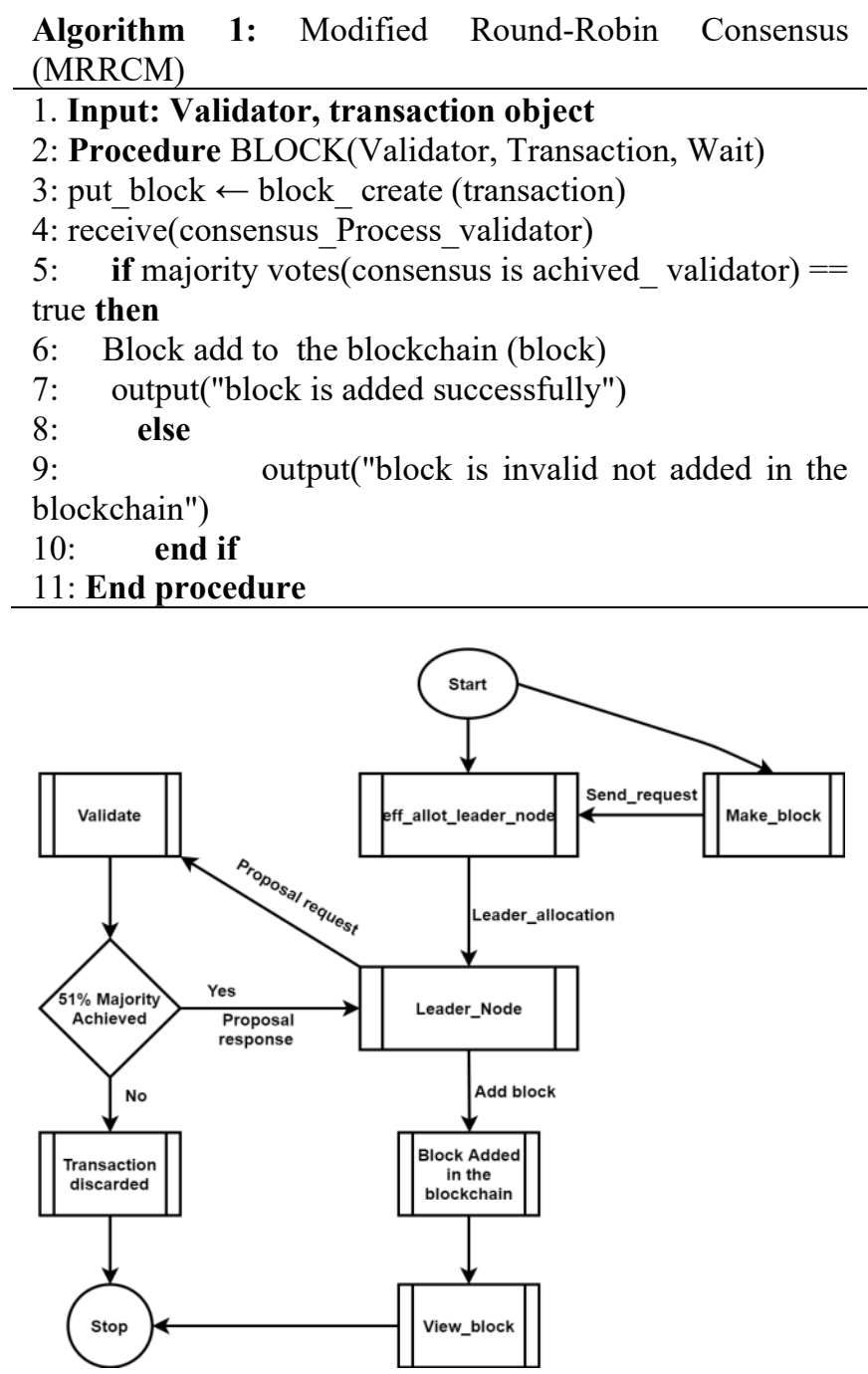

Figure 2. Modified round-robin consensus process flow
The proposed mechanism aims to develop a property registry system based on DLT and optimal query algorithms (discussed in section 4) for querying the ledger. With this querying algorithm, we can to obtain a history of the transactions based on any attribute. Therefore all registry offices in the state willing to deploy the proposed system must join the proposed blockchain-based framework to provide a fast and optimal query algorithm for querying the ledger and registration process to seller/buyer. The following are the different modules of the proposed framework:

-Validate Transaction Module

-Add a block to the blockchain

-View block Module

\subsection{Validate transaction module}

This module will validate the transaction among the network. For example, if the land property registration details exist in the blockchain, it will update user registry data. There may be a case of a faulty transaction. In this transaction, let us suppose there is no property registered with the property record number mentioned in the transaction. Also, there may be transactions wherein the seller or buyers are not registered to the application shown in Figure 3. Such trades are invalidated.
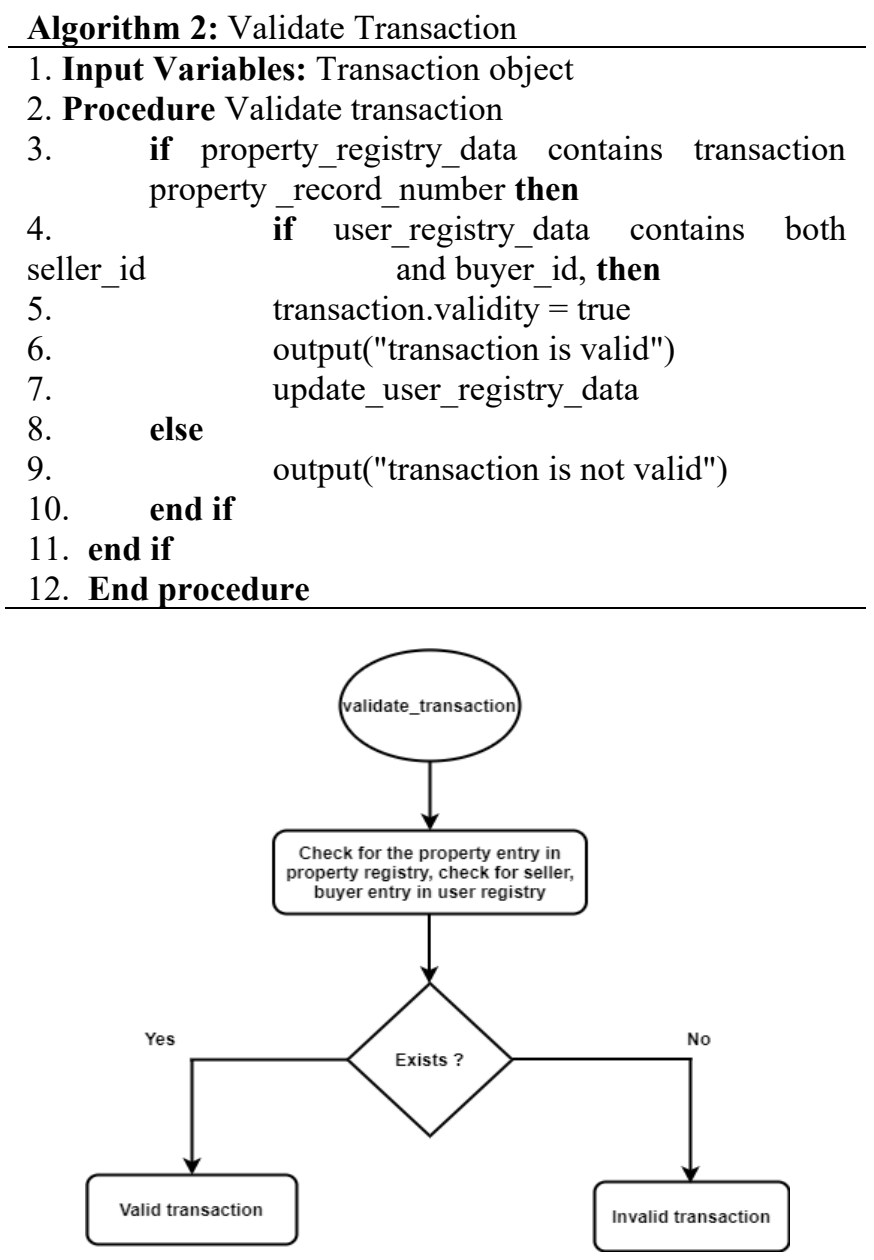

Figure 3. Validate transaction module

\subsection{Add a block to the Blockchain}

In this step, the module will add a block to the blockchain as per algorithm 3 . The block contains the timestamp of block 
creation and a hash to the previous block. Once all transactions are validated, the block will be added to the blockchain; otherwise, the transaction is invalid and discarded (shown in Figure 4).
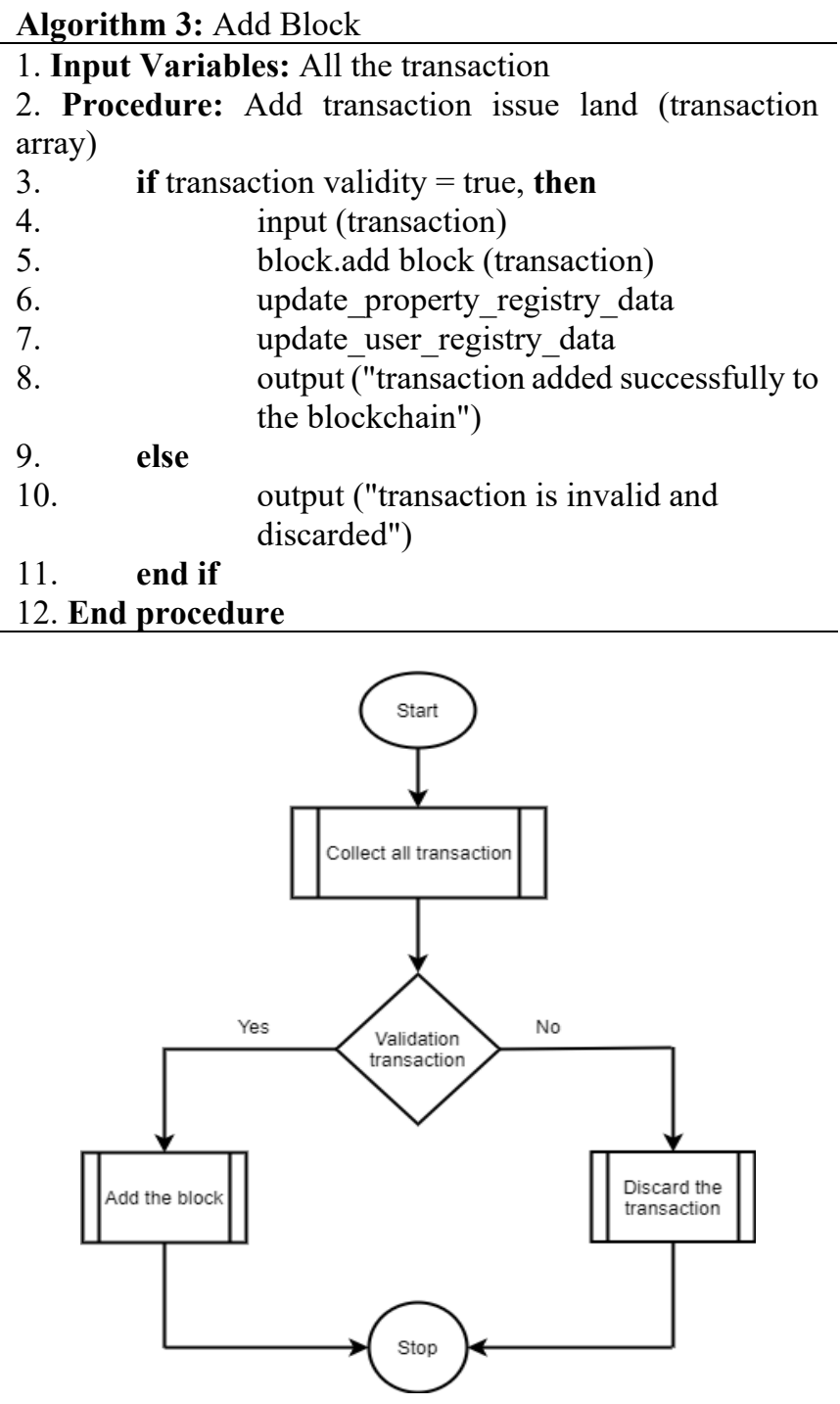

Figure 4. Add block module

\subsection{View block module}

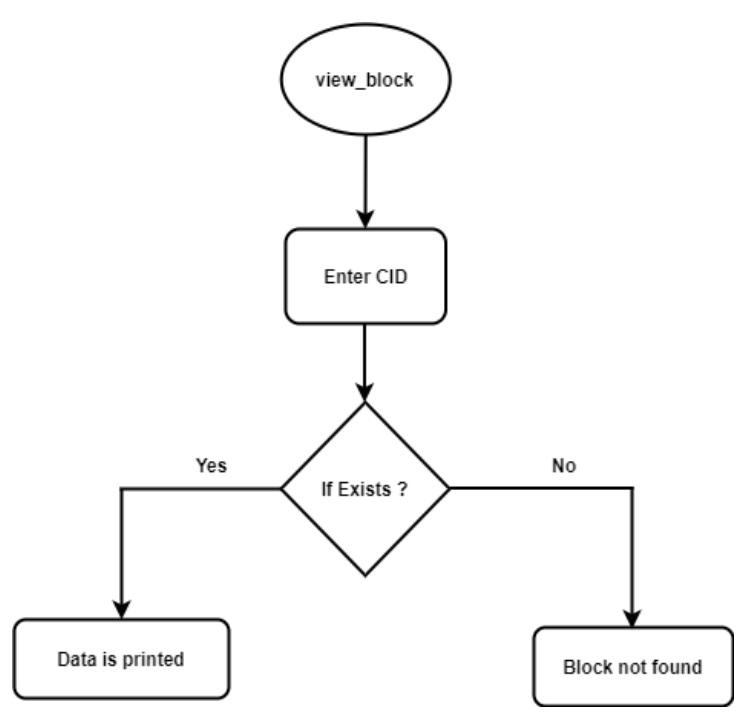

Figure 5. View block module
This module is for viewing the block with the block's Content Identifier (CID) as per algorithm 4. If CID exists in blockchain, then block data will be shown. Otherwise, the block does not exist in the blockchain (shown in Figure 5).

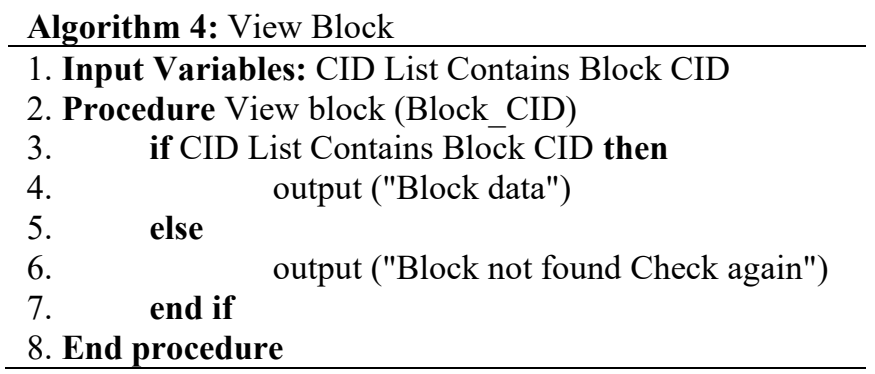

\section{QUERY LEDGER MODULE}

In this section, three different searching algorithms are discussed as follows.

-Extensive Linear Search

-Hash Table based Approach

-Optimized Hash Table Approach

\section{(1) Extensive linear search}

A list of attributes/CID is being maintained in a file for all the blocks added to the network. According to the basic concept of linear search, all the blocks are parsed to find out the transaction, which has the attribute/CID value equal to the searching key value as per algorithm 5. If CID exists in blockchain, then block data will be shown. Otherwise, the block does not exist in the blockchain (shown in Figure 6).

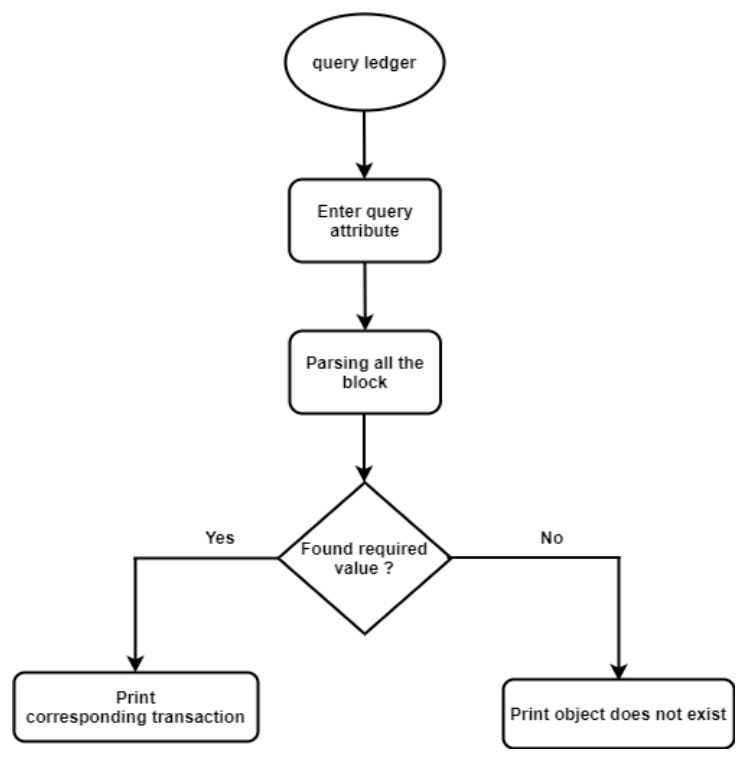

Figure 6. Query Ledger Linear Module

Algorithm 5: Query Ledger

1. Input Variables: Attribute to be queried on and value of the attribute

2. Procedure Query ledger (query_attribute, attribute_value)

3. Loop through all the CIDS

4. Parse all the block

5. Loop through all the transaction in the particular block

6. if block.transaction.attribute.value $==$ attribute then 


\begin{tabular}{lll}
\hline 7. & output("Block Content Identifier") \\
8. & else & \\
9. & output("Value not found") \\
10. & end if & \\
11. End procedure \\
\hline
\end{tabular}

\section{(2) Hash table based approach}

In this approach, a hash table is been maintained for each attribute. Whenever we are querying for a particular attribute, the searching key is will be searched in the hash. If the searching key is found in the hash table then the corresponding block details will be displayed or printed. If the searching key doesn't exist in the hash table, it means that the value is not found as per algorithm 6 (shown in Figure 7).

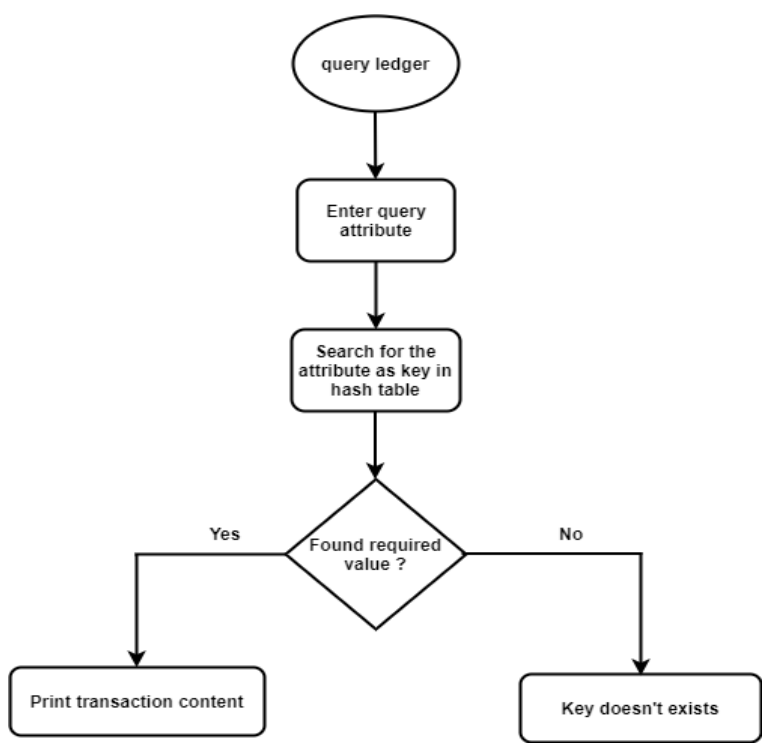

Figure 7. Query ledger hash table module

Algorithm 6. Query Ledger Hash Table Based
1. Input Variables: Attribute to be queried on and value of
the attribute
2. Procedure Query ledger(query_attribute,
attribute_value)
3. Check the hash table for the query attribute
4. if attribute.hash_table_contains_attribute_value
then output("All the content identifiers and
$5 . \quad$ are printed")
transaction identifiers are

\begin{tabular}{l}
\hline $6 . \quad$ else \\
$7 . \quad$ output("Value not found") \\
$8 . \quad$ end if \\
9. End procedure \\
\hline
\end{tabular}

\section{(3) Optimized hash table approach}

This approach is similar to the hash-based method. We will be mapping the blocks and the corresponding transactions with the help of a Merkle DAG. Mapping takes place during the addition of the block itself. A block is mapped to another block only if they have common attribute values that can be queried upon. This approach will show improvement as there is a direct mapping between blocks without traversing all the blocks. Once the mapping is obtained, we can check the hash table for the retrieved blocks.

\section{ATTRIBUTES OF PROPERTY TRANSACTION IN BLOCK}

This registry of the database is used to maintain the details of the registered properties. Every time when property ownership is transferred, the property's record is updated, and the transaction is added to the shared ledger. The approval organizations endorse the transaction for adding the property to the asset registry of properties. This transaction, once validated, is generated as a block that is added to the blockchain. The data is stored in text format. Table 1 illustrates the sample property (Summary File) registry of the data being stored. The attributes of the property registry are:

Property Number: This is a unique identification number for the land registered.

Property Location: This is the location of the property.

Property E Stamp number: This is the permanent address of the individual.

Property E Stamp Issue Date: This represents the issue date of the property.

Property Owner: This gives the property owner's name.

Owner Postal Code: This gives the postal code of the property owner.

Owner Address: This specifies the address of the owner.

Owner Postal Code: This specifies the postal code of the owner.

Owner City: This specifies the city of the owner.

Owner State: This specified the state of the owner.

Table 1. Property transaction record

\begin{tabular}{|c|c|c|c|c|c|c|c|c|c|}
\hline $\begin{array}{l}\text { Property } \\
\text { Number }\end{array}$ & $\begin{array}{l}\text { Property } \\
\text { Location }\end{array}$ & $\begin{array}{c}\text { Property } \\
\text { Area }\end{array}$ & $\begin{array}{l}\text { Property e- } \\
\text { stamp } \\
\text { number } \\
\end{array}$ & $\begin{array}{c}\text { Property e- } \\
\text { stamp issue } \\
\text { date }\end{array}$ & $\begin{array}{c}\text { Property } \\
\text { owner }\end{array}$ & $\begin{array}{c}\text { Owner } \\
\text { postal } \\
\text { code }\end{array}$ & $\begin{array}{c}\text { Owner } \\
\text { address }\end{array}$ & $\begin{array}{c}\text { Owner } \\
\text { city }\end{array}$ & $\begin{array}{c}\text { Owner } \\
\text { state }\end{array}$ \\
\hline 101 & Lucknow & Ashiyana & 12345 & $12 / 09 / 19$ & Anvay & 226012 & $\begin{array}{l}\text { LDA } \\
\text { Colony }\end{array}$ & Lucknow & U.P. \\
\hline 102 & Allahabad & Teliarganj & 13423 & $17 / 05 / 18$ & Anurag & 211004 & MNNIT & Allahabad & U.P. \\
\hline 103 & Kanpur & Rama Devi & 18746 & $15 / 01 / 15$ & Khushi & 208001 & Sector 2 & Kanpur & U. P. \\
\hline 104 & Delhi & Rohini & 10845 & $20 / 07 / 96$ & Mitra & 110001 & Sector 5 & Delhi & Delhi \\
\hline 105 & Ghaziabad & Patel Nagar & 16091 & $10 / 08 / 90$ & Ayushi & 201003 & $\begin{array}{c}\text { Patel } \\
\text { Nagar }\end{array}$ & Ghaziabad & U. P. \\
\hline 106 & Allahabad & Allahabad & 200796 & $20 / 07 / 96$ & Niharika & 211004 & Allahabad & Allahabad & U.P. \\
\hline 107 & Lucknow & Malihabad & 231568 & 08/09/1975 & Suman & 226102 & Malihabad & Lucknow & U.P. \\
\hline 108 & Ghaziabad & Noida & 556661 & $12 / 05 / 2002$ & Anvi & 201003 & Noida & Noida & U.P. \\
\hline 109 & Delhi & Lucknow & 54698 & $20 / 08 / 20$ & Lavi & 226012 & Lucknow & Ashiyana & U.P. \\
\hline 110 & Allahabad & Teliyarganj & 77956 & 20/02/1990 & Sonali & 211004 & Allahabad & Allahabad & U.P. \\
\hline
\end{tabular}


Table 2. User transaction record

\begin{tabular}{cccccc}
\hline Identification Number & Property Owner & Owner Postal Code & Owner Address & Owner City & Owner State \\
\hline 101 & Anvay & 226012 & LDA Colony & Lucknow & U. P. \\
102 & Anurag & 211004 & MNNIT & Allahabad & U. P. \\
103 & Khushi & 208001 & Sector 2 & Kanpur & U. P. \\
104 & Mitra & 110001 & Sector 5 & Delhi & Delhi \\
105 & Ayushi & 201003 & Patel Nagar & Ghaziabad & U. P. \\
106 & Niharika & 211002 & Allahabad & Allahabad & U. P. \\
107 & Suman & 226102 & Malihabad & Lucknow & U. P. \\
108 & Anvi & 201003 & Noida & Noida & U. P. \\
109 & Lavi & 226012 & Lucknow & Ashiyana & U. P. \\
110 & Sonali & 211004 & Allahabad & Allahabad & Delhi. \\
\hline
\end{tabular}

This asset registry of the database is used to maintain the details of the registered users. When a user sells/buys a property, the user's record is added to the user asset registry once the transaction is approved. The approval organizations validate the transaction for adding the user. This transaction, once validated, is generated as a block that is added to the blockchain. Table 2 illustrates the sample property (Summary File) asset of the data being stored. The attributes of the user registry are following:

User Name: This gives the property user's name.

User Postal Code: This gives the postal code of the property owner.

User's Address: This specifies the address of the user.

User's Postal Code: This specifies the postal code of the user.

User's City: This specifies the city of the user.

User's State: This specified the state of the user.

The data is stored in text format. The unique identification number of this registered property is the user identification number. To query the registered property, this unique id shall be used. If any attempt is made to register a user with a previously existing identification number, then the system will generate a message that the user already exists.

\section{RESULT AND ANALYSIS}

The proposed model Indian Land Registry System successfully runs on multiple nodes. The proposed algorithm MRRCM is compared with the proof-of-work and round-robin approach. The modified hash table's searching time is compared to the extensive liner search and hash table search approach.

\subsection{Comparison with proof-of-work and round-robin}

The proposed approach MRRCM execution time is compared with PoW, and Round-robin approaches. Table 3 shows the execution time PoW, Round-robin, and MRRCM. The number of nodes is varying from 05-50.

Table 3. Time taking of execution of block

\begin{tabular}{ccccccc}
\hline $\begin{array}{c}\text { S. } \\
\text { No. }\end{array}$ & $\begin{array}{c}\text { Number of } \\
\text { Nodes }\end{array}$ & PoW & $\begin{array}{c}\text { Round- } \\
\text { robin }\end{array}$ & MRRCM & $\begin{array}{c}\text { Comparative difference PoW \& } \\
\text { MRRCM }\end{array}$ & $\begin{array}{c}\text { Comparative difference Round-robin } \\
\text { \& MRRCM }\end{array}$ \\
\hline 1 & 5 & 58.9 & 2.46 & 1.54 & 95.75 & 0.92 \\
2 & 10 & 129.8 & 2.98 & 1.98 & 98.47 & 1.00 \\
3 & 15 & 154.4 & 4.62 & 2.60 & 98.44 & 2.02 \\
4 & 20 & 186.2 & 5.22 & 3.05 & 98.36 & 2.17 \\
5 & 30 & 207.6 & 9.85 & 7.12 & 96.65 & 2.46 \\
6 & 40 & 242.8 & 16.85 & 12.58 & 94.81 & 4.27 \\
7 & 50 & 296.4 & 28.84 & 20.92 & 92.94 & 8.64 \\
\hline
\end{tabular}

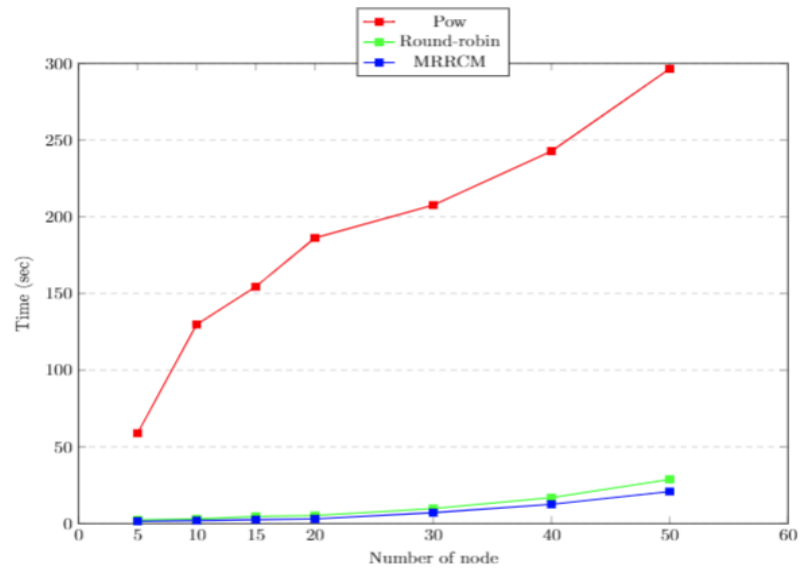

Figure 8. Comparison number of block generated by PoW, round-robin, and MRRCM

Figure 8 shows the graphical view of comparative analysis of PoW, Round-robin, and MRRCM approach in terms of the number of the generated blocks. The number of node range from 05 to 50. The proposed MRRCM approach reduces the block generation time on an average by $3.06 \%$ and $96.48 \%$ compared to the round-robin approach and PoW approach, respectively.

\subsection{Comparison based on block search time}

In this article, the proposed modified hash table approach is successfully implemented as a query system in the IPFS network. The blockchain of nodes varying from 10 to 350 blocks is considered. The application gives query results based on any attribute from the list of specified attributes by the user. Efforts were put to reduce the load on the existing system during the manual execution of the application. The performance of the linear search is the slowest among all the algorithms implemented. The hash table and the modified hash table algorithm provide the best query and search on the blockchain compared to linear search, as shown in Table 4. 
Table 4. Time taken for searching attribute

\begin{tabular}{|c|c|c|c|c|c|c|c|c|}
\hline $\begin{array}{l}\text { Sr. } \\
\text { No. }\end{array}$ & $\begin{array}{l}\text { Number } \\
\text { of Nodes }\end{array}$ & $\begin{array}{c}\text { Extensive } \\
\text { Liner } \\
\text { Search } \\
\text { (msec) }\end{array}$ & $\begin{array}{c}\text { Hash } \\
\text { Table } \\
\text { Search } \\
(\mathrm{msec})\end{array}$ & $\begin{array}{c}\text { Modified } \\
\text { Hash } \\
\text { Table } \\
\text { (msec) }\end{array}$ & $\begin{array}{c}\text { Diff. b/w } \\
\text { Extensive } \\
\text { Liner Search } \\
\text { \& Modified } \\
\text { Hash Table } \\
\text { (msec) }\end{array}$ & $\begin{array}{c}\text { Diff. b/w } \\
\text { Extensive } \\
\text { Liner Search } \\
\text { \& Modified } \\
\text { Hash Table } \\
\text { (msec) }\end{array}$ & $\begin{array}{c}\text { Modified Hash } \\
\text { Table } \\
\text { improvement (\%) } \\
\text { wrt Extensive } \\
\text { Liner Search }\end{array}$ & $\begin{array}{c}\text { Modified Hash } \\
\text { Table } \\
\text { improvement (\%) } \\
\text { wrt Hash Table } \\
\text { Search }\end{array}$ \\
\hline 1 & 10 & 13.60 & 7.40 & 5.79 & 7.81 & 1.60 & 57.42 & 21.75 \\
\hline 2 & 20 & 14.39 & 7.82 & 6.11 & 8.29 & 1.72 & 56.78 & 21.99 \\
\hline 3 & 30 & 15.59 & 7.99 & 6.98 & 8.91 & 0.84 & 57.15 & 10.51 \\
\hline 4 & 50 & 16.45 & 8.15 & 7.50 & 8.95 & 0.65 & 54.40 & 7.97 \\
\hline 5 & 100 & 18.23 & 8.83 & 7.80 & 10.43 & 1.03 & 57.21 & 11.66 \\
\hline 6 & 150 & 19.49 & 9.45 & 7.94 & 11.55 & 1.51 & 59.49 & 15.97 \\
\hline 7 & 200 & 20.98 & 10.13 & 8.12 & 12.86 & 2.01 & 61.29 & 19.84 \\
\hline 8 & 250 & 22.45 & 11.10 & 8.53 & 13.92 & 2.57 & 62 & 23.15 \\
\hline 9 & 300 & 24.93 & 12.16 & 8.98 & 15.95 & 3.18 & 63.97 & 26.15 \\
\hline 10 & 350 & 27.22 & 13.10 & 9.45 & 17.77 & 3.65 & 65.28 & 27.86 \\
\hline
\end{tabular}

Figure 9 shows the graphical representation of the comparative assessment of the modified hash table, existing hash table, and extensive liner search algorithm. The number of nodes range from 10 to 350 . The proposed approach modified the hash table thereby reducing the search time on an average by $18.68 \%$ as compared to the hash table search approach. The searching time is drastically reduced by $59.49 \%$ as compared to the extensive liner search. It shows that the modified hash search requires less time than the extensive liner searches and hash table search approach.

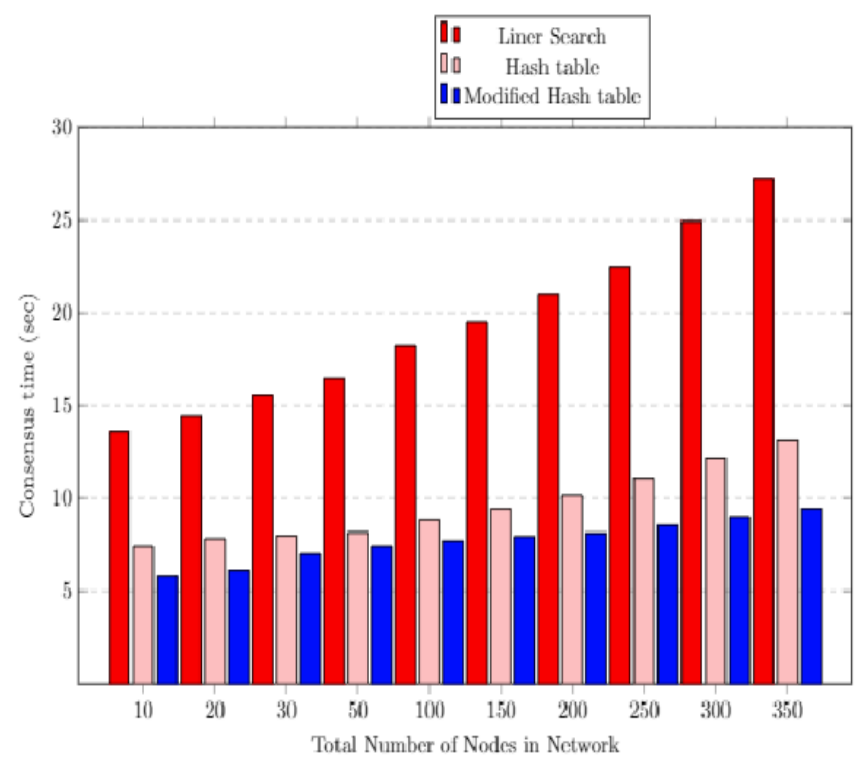

Figure 9. Comparison searching by the liner search, hash table search, and modified hash table

\section{CONCLUSION AND FUTURE DIRECTION}

This article proposes a blockchain-based framework to provide scalable, tamper-proof, focused on an overview of DLT and its use in the Land Registry Management System (LRMS). This article aims to implement a registry of land using blockchain technology, optimize its querying process and provide a secure, transparent, fast access, and decentralized framework. Interplanetary File System (IPFS) provides an infrastructure that offers a precise portrayal of all the members' roles. The application interacts with the blockchain network, which is built using IPFS. It proposes a scalable framework as the proposed consensus algorithm MRRCM achieves less time required to generated a block on an average by $3.06 \%$ than the round-robin approach and $96.48 \%$ compared to the PoW approach. The article also implements a land registry modified hash table search algorithm using blockchain technology and optimizes its querying process. Modified hash table search algorithm reduces the search time on an average by $18.68 \%$ as compared to the hash table and by $59.49 \%$ compared to the extensive linear search approach. Future work will include reducing blockchain overhead and improving search functions, e.g., similarity queries, boolean queries, and so on. Also, we will consider conjunctive keyword search.

\section{REFERENCES}

[1] Singh, N., Vardhan, M. (2019). Distributed ledger technology based property transaction system with support for IoT devices. International Journal of Cloud Applications and Computing (IJCAC), 9(2): 60-78. https://doi.org/10.4018/IJCAC.2019040104

[2] DILRMP: Digital India Land Records Modernization Programme (2008). Government of India. http://dilrmp.nic.in.

[3] Thakur, V., Doja, M.N., Dwivedi, Y.K., Ahmad, T., Khadanga, G. (2020). Land records on blockchain for implementation of land titling in India. International Journal of Information Management, 52: 101940. https://doi.org/10.1016/j.ijinfomgt.2019.04.013

[4] Nakamoto, S. (2019). Bitcoin: A peer-to-peer electronic cash system. Manubot.

[5] Zheng, Z., Xie, S., Dai, H., Chen, X., Wang, H. (2017). An overview of blockchain technology: Architecture, consensus, and future trends. 2017 IEEE International Congress on Big Data (BigData Congress), Honolulu, HI, USA, pp. 557-564. https://doi.org/10.1109/BigDataCongress.2017.85

[6] https://www.factom.com/.

[7] Tschorsch, F., Scheuermann, B. (2016). Bitcoin and beyond: A technical survey on decentralized digital currencies. IEEE Communications Surveys \& Tutorials, 18(3): 2084-2123. https://doi.org/10.1109/COMST.2016.2535718

[8] https://www.teriin.org/eventdocs/files/Case-StudyVishakhapatnam.pdf. 
[9] Singh, K., Singh, N., Kushwaha, D.S. (2018). An interoperable and secure e-wallet architecture based on digital ledger technology using blockchain. 2018 International Conference on Computing, Power and Communication Technologies (GUCON), Greater Noida, India, pp. 165-169. https://doi.org/10.1109/GUCON.2018.8674919

[10] Kumar, A., Yadav, A.S., Kushwaha, D.S. (2020). VChain: Efficient blockchain based vehicular communication protocol. 2020 10th International Conference on Cloud Computing, Data Science \& Engineering (Confluence), Noida, India, pp. 762-768. https://doi.org/10.1109/Confluence47617.2020.905780

[11] Singh, N., Vardhan, M. (2019). Digital ledger technology-based real estate transaction mechanism and its block size assessment. International Journal of Blockchains and Cryptocurrencies, 1(1): 67-84. https://doi.org/10.1504/IJBC.2019.101853

[12] Singh, N., Vardhan, M. (2018). Blockchain based Estamp procurement system with efficient consensus mechanism and fast parallel search. Journal of Mechanics of Continua and Mathematical Sciences, 13(4):

73-89. https://doi.org/10.26782/jmcms.2018.10.00007

[13] Mallick, S., Kushwaha, D.S. (2012). An efficient publish-subscribe protocol for collaborative content delivery-ex-PUSH. 2012 International Symposium on Cloud and Services Computing, Mangalore, India, pp. 152-156. https://doi.org/10.1109/ISCOS.2012.27

[14] Singh, N., Vardhan, M. (2021). Multi-objective optimization of block size based on CPU power and network bandwidth for blockchain applications. Proceedings of the Fourth International Conference on Microelectronics, Computing and Communication Systems, Singapore, $\quad$ pp. 69-78. https://doi.org/10.1007/978-981-15-5546-6 6

[15] Singh, N., Kumar, T., Vardhan, M. (2019). Blockchain based e-cheque clearing framework. Scalable Computing: Practice and Experience, 20(3): 511-526. https://doi.org/10.12694/scpe.v20i3.1506

[16] Singh, N., Kumar, T., Vardhan, M. (2020). Blockchainbased e-cheque clearing framework with trust based consensus mechanism. Cluster Computing, 1-15. https://doi.org/10.1007/s10586-020-03163-6

[17] Singh, N., Vardhan, M. (2020). Computing Optimal Block Size for Blockchain based Applications with Contradictory Objectives. Procedia Computer Science, 171: 1389-1398. https://doi.org/10.1016/j.procs.2020.04.149

[18] Chromaway. (2018). Future house purchases. Chromaway. https://chromaway.com/ landregistry/.

[19] Yadav, A.S., Shikha, S., Gupta, S., Kushwaha, D.S. (2021). The efficient consensus algorithm for land record management system. IOP Conference Series: Materials Science and Engineering, 1022(1): 012090. https://doi.org/10.1088/1757-899X/1022/1/012090

[20] Nguyen, G.T., Kim, K. (2018). A survey about consensus algorithms used in blockchain. Journal of Information Processing Systems, 14(1): 101-128. https://doi.org/10.3745/JIPS.01.0024

[21] Gupta, S., Sinha, S., Bhushan, B. (2020). Emergence of blockchain technology: Fundamentals, working and its various implementations. Working and its Various Implementations. http://dx.doi.org/10.2139/ssrn.3569577

[22] Kiayias, A., Russell, A., David, B., Oliynykov, R. (2017, August). Ouroboros: A provably secure proof-of-stake blockchain protocol. In Annual International Cryptology Conference, pp. 357-388. https://doi.org/10.1007/978-3319-63688-7_12 